\title{
Thermodynamic solvation of a series of homologous $\alpha$-amino acids in non-aqueous mixture of ethylene-glycol and $\mathbf{N}, \mathbf{N}$-dimethyl formamide
}

\author{
Kalachand Mahali ${ }^{1}$, Sanjay Roy ${ }^{2}$, Bijoy Krishna Dolui ${ }^{{ }^{*}}$ \\ ${ }^{1}$ Department of Chemistry, Visva-Bharati, Santiniketan, India; ${ }^{*}$ Corresponding Author: bijoy_dolui@yahoo.co.in \\ ${ }^{2}$ Department of Chemistry, Shibpur Dinabundhoo Institution (College), Howrah, India.
}

Received 3 March 2011; revised 4 July 2011; accepted 20 July 2011.

\begin{abstract}
Standard free energies $\left(\Delta G_{t}^{0}(i)\right)$ and entropies $\left(\Delta S_{t}^{0}(i)\right)$ of transfer of some homologous $\alpha$-amino acids viz. glycine (gly), Dl-alanine (ala), Dl- $\alpha$-amino butyric acid (aba) and DI-nor-valine (nor-val) from protic ethylene glycol (EG) to dipolar aprotic $\mathrm{N}, \mathrm{N}$-dimethyl formamide (DMF) have been evaluated from solubility measurements at five equidistant temperatures i.e. from $15^{\circ} \mathrm{C}$ to $35^{\circ} \mathrm{C}$. The observed $\Delta G_{t}^{0}(i)$ and $\mathrm{T} \Delta S_{t}^{0}(i)$ vs composition profiles are complicated because of the various interaction effects. The chemical effects of the transfer Gibbs energies $\left(\Delta G_{t, c h}^{0}(i)\right)$ and entropies of transfer $\left(T \Delta S_{t, c h}^{0}(i)\right)$ have been obtained after elimination of cavity effect, estimated by the scaled particle theory and dipole-dipole interaction effects, estimated by the use of Keesom-orientation expression. The chemical contributions of transfer energetics of homologous $\alpha$-amino acids are guided by the composite effects of increased dispersion interaction, basicity and decreased acidity, hydrogen bonding effects and solvophobic solvation of ethylene glycol and $\mathbf{N}, \mathbf{N}$-dimethyl formamide mixed solvent as compared to that of reference solvent (ethylene glycol).
\end{abstract}

Keywords: Non-aqueous Solvent System; Transfer Energetic; Zwitterions; $\alpha$-Amino Acids; Solvophobic Solvation

\section{INTRODUCTION}

It is well known that amino acids are fundamental structural units of proteins. The native state of a protein is determined by the nature and sequence of its constituent's amino acids as well as by the solvent environment.
Much attention had been paid [1-5] to determine the various thermodynamic properties of amino acids in aqua-organic mixed solvent system.

The purpose of such studies is to gain the various aspects of protein folding and unfolding processes and protein hydration [6,7]. In this regard Tanford, Nozaki and other authors $[8,9]$ reported free energies of some amino acids from water to urea from solubility measurements. Transfer free energies and entropies data of some amino acids, dipeptides, tripeptides, and other biomolecules in aqueous ethylene glycol and glycerol are also available [10-13].

All these experiments tried to give an idea about the relative stabilization of those amino acids and other biomolecules in aqua-organic media with respect to water and the complex solute-solvent and solvent-solvent interactions therein.

In fact, the environment in which the different biological processes occur may be much more "amide like" than "water like". Therefore relevant data in amide solvents like $N, N$-dimethyl formamide in particular, are likely to be very much useful to understand biological processes better [14].

Also if we want to understand the role of the highly complex aqueous chemistry in the context of stabilizations of proteins and other bimolecules composed of amino acids and the involved structural "eccentricities" of water we have to first realize the chemistry of much similar non-aqueous solvents as a baseline "normal behavior", there by a better understanding of solute-solvent interactions will be possible in aqua-organic solvents.

With that end in view, in the present paper we are reporting the transfer free energies $\left(\Delta G_{t}^{0}(i)\right)$ and entropies $\left(\Delta S_{t}^{0}(i)\right)$ of a series of homologous $\alpha$-amino acids, namely glycine (gly), DL-alanine (ala), DL- $\alpha$-aminobutyric acids (aba) and DL-Nor-valine (val) from ethylene glycol (EG) to non-aqueous mixture of protic ethylene-glycol and dipolar aprotic $N, N$,-dimethyl formamide 
(ethylene glycol and $\mathrm{N}, \mathrm{N}$-dimethyl formamide) at $25^{\circ} \mathrm{C}$, as determined from solubility measurements using "formal titrimetry" at five equidistant temperatures ranging from $15^{\circ} \mathrm{C}-35^{\circ} \mathrm{C}$.

After eliminating effects due to cavity formation and dipole-dipole interactions and neglecting dipole-induced dipole interactions the results have been discussed in terms of dispersion interaction, acidity-basicity, solvophilic and solvophobic solvation and in the case of transfer entropies in terms of relative structuredness as well.

\section{MATERIALS AND METHODS}

\subsection{Materials}

$\alpha$-amino acids like glycine (gly) (E Merck) and Dlalanine (ala) ,amino butyric acid (aba) and nor-valine (n-val) were used after drying as described earlier [15].

Ethylene glycol (LR, BDH) was purified by the usual method [16]. Ethylene glycol (LR, BDH) was refluxed with $2 \%-3 \% \mathrm{NaOH}$ (Merck) fpr $3-4$ hours and then distilled; the distilled glycol was then dried over freshly baked anhy. $\mathrm{Na}_{2} \mathrm{SO}_{4}$ (Merck) for 4 - 5 days, then decanted off and fractionally distilled through a $2 / 3 \mathrm{~m}$ long vigreux column, rejecting the head and tail portions.

$N, N$-dimethyl formamide (DMF) (LR, BDH) was purified [16] first by distilling under reduced pressure in $\mathrm{N}_{2}$ atmosphere and preserving the distillate over dry $\mathrm{K}_{2} \mathrm{CO}_{3}$ (Merck) for a week or so. The solvent was then decanted off and treated with pure $\mathrm{P}_{2} \mathrm{O}_{5}$ (Riedel) and finally distilled under reduced pressure.

The water content of the solvents were determined by Karl-Fisher titration and found to be less than 0.02-mol $\mathrm{dm}^{-3}$ in each case.

Non-aqueous mixtures of co-solvent (ethylene glycol and $N, N$-dimethyl formamide) that have been used were $20,40,60,80$ and $100 \mathrm{wt} \%$ and were protected by storing in desiccators when not in use.

\subsection{Methods}

The solubility of these four amino acids were measured by the formol titrimetric method as described in our previous paper $[12,17]$. These measurements were taken at $15^{\circ} \mathrm{C}, 20^{\circ} \mathrm{C}, 25^{\circ} \mathrm{C}, 30^{\circ} \mathrm{C}$ and $35^{\circ} \mathrm{C}$ temperatures. The low-cum- high temperature thermostat used for all measurements was capable of registering temperatures having an accuracy of $\pm 0.1^{\circ} \mathrm{C}$. Three sets of measurements were made for all the solutes by equilibrating the solutions from both above and below the required temperatures and at least two sets of measurements were made for all the solvents and the solubilities were found to agree to within $\pm 1 \%$ to $1.5 \%$.

\section{RESULTS}

\subsection{Computation of Total Transfer Free Energy and Entropy}

The solvent parameters are listed in Table 1. The measured solubility $(\mathrm{m})$ of the amino acids (on molal scale) is listed in Table 2.

As in the previous studies by Bates and coworkers on Tris [18] and by Kundu and coworkers [18,19] on nonelectrolyte like para-nitroaniline, benzoic acid and amino acids [15], glycine (G), diglycine (DG), and triglycine (TG), the Gibbs energies of solutions $\left(\Delta G_{s}^{0}\right)$ of these amino acids on molal scale were calculated for each solvent using Eq.1.

$$
\Delta G_{s}^{0}=-R T \ln C y=-R T \ln C=-R T \ln m
$$

where $y$ is the molar activity coefficient of the solutes but taken tentatively to be unity in each solvent. True, since these amino acids are likely to be mostly in zwitterionic forms as in non-aqueous solvent mixtures [20, 21], the involved activity coefficient factor-RTlny in $\Delta G_{s}^{0}$ arising from interactions of dipolar solutes with large dipole moments may not be that small. But as there is neither the required experimental data nor any appropriate theoretical correlations for computing the same, these have been tacitly taken to be negligibly small, as is usually done for non-electrolytes [11]. This is because the effective contribution of activity coefficient factor $-R T \ln y_{s} / y_{R}$ in the transfer free energetics $\Delta G_{t}^{0}(i)=$ $\Delta G_{s}^{0}(i)-\Delta G_{R}^{0}(i)$ in particular which is our main concern likely to be hardly significant.

The free energies, $\Delta G_{s}^{0}$ at different temperatures are fitted by the method of least squares to an equation of the form;

$$
\Delta G_{s}^{0}=a+b T+c T \ln T
$$

where $T$ is the temperature in Kelvin scale. The values of the coefficients $a, b, c$ are presented in Table 3 . These are found to reproduce the experimental data within \pm $0.04 \mathrm{~kJ} \cdot \mathrm{mol}^{-1}$. Transfer Gibbs energies, $\Delta G_{t}^{0}$ and entropies, $\Delta S_{t}^{0}$ of the amino acids from ethylene-glycol to $\mathrm{N}, \mathrm{N}$-dimethylformamide mixtures were calculated at $25^{\circ} \mathrm{C}$ on mole fraction scale by using the following Eqs.3 \& 4:

$$
\begin{gathered}
\Delta G_{t}^{0}(i)={ }_{s} \Delta G_{s}^{0}(i)-{ }_{R} \Delta G_{s}^{0}(i) \\
\Delta G_{t}^{0}(i)=\left(a_{s}-a_{R}\right)+\left(b_{s}-b_{R}\right) T \\
+\left(c_{s}-c_{R}\right) T \ln T-R T \ln \left(M_{s} / M_{R}\right)
\end{gathered}
$$

and

$$
\Delta S_{t}^{0}(i)=\left(b_{R}-b_{s}\right)+\left(c_{R}-c_{s}\right)(1+\ln T)+R \ln \left(M_{s} / M_{R}\right)
$$


Table 1. Solvent parameters i.e. values of mean molecular weight $\left(M_{s}\right)$, density $\left(d_{s}\right)$, dielectric constant $\left(\varepsilon_{s}\right)$ of the ethylene glycol and $N, N$-dimethyl formamide system at different temperatures.

\begin{tabular}{|c|c|c|c|c|}
\hline $\begin{array}{l}\text { wt \% } \\
\text { DMF }\end{array}$ & $\mathbf{x}_{\mathrm{DMF}}$ & $\underset{\left(\mathbf{k g} \cdot \mathbf{m}^{-3}\right)}{\mathbf{M}_{\mathrm{S}}}$ & $10^{3} d_{s}$ & $\varepsilon_{\mathrm{s}}$ \\
\hline & & $15^{\circ} \mathrm{C}$ & & \\
\hline 0 & 0 & 62.07 & 1.1159 & 42.64 \\
\hline 20 & 0.175 & 63.92 & 1.0815 & 41.92 \\
\hline 40 & 0.362 & 65.98 & 1.0497 & 41.14 \\
\hline 60 & 0.561 & 68.17 & 1.0152 & 40.32 \\
\hline 80 & 0.744 & 70.51 & 0.9849 & 39.44 \\
\hline \multirow[t]{2}{*}{100} & 1.000 & 73.14 & 0.9500 & 37.64 \\
\hline & & $20^{\circ} \mathrm{C}$ & & \\
\hline 0 & 0 & 62.07 & 1.1150 & 41.67 \\
\hline 20 & 0.175 & 63.92 & 1.0750 & 40.96 \\
\hline 40 & 0.362 & 65.98 & 1.0450 & 40.20 \\
\hline 60 & 0.561 & 68.17 & 1.0125 & 39.39 \\
\hline 80 & 0.744 & 70.51 & 0.9740 & 37.50 \\
\hline \multirow[t]{2}{*}{100} & 1.000 & 73.14 & 0.9500 & 37.64 \\
\hline & & $25^{\circ} \mathrm{C}$ & & \\
\hline 0 & 0 & 62.07 & $\begin{array}{c}1.1099 \\
(1.1100)\end{array}$ & 40.70 \\
\hline 20 & 0.175 & 63.92 & 1.0750 & 39.90 \\
\hline 40 & 0.362 & 65.98 & 1.0410 & 39.10 \\
\hline 60 & 0.561 & 68.17 & 1.0070 & 38.30 \\
\hline 80 & 0.744 & 70.51 & 0.9740 & 37.50 \\
\hline \multirow[t]{2}{*}{100} & 1.000 & 73.14 & $\begin{array}{c}0.9443 \\
(0.9440)\end{array}$ & 36.70 \\
\hline & & $30^{\circ} \mathrm{C}$ & & \\
\hline 0 & 0 & 62.07 & 1.1050 & 39.73 \\
\hline 20 & 0.175 & 63.92 & 1.0700 & 39.04 \\
\hline 40 & 0.362 & 65.98 & 1.0350 & 38.32 \\
\hline 60 & 0.561 & 68.17 & 1.0050 & 37.53 \\
\hline 80 & 0.744 & 70.51 & 0.9750 & 36.69 \\
\hline \multirow[t]{2}{*}{100} & 1.000 & 73.14 & 0.9400 & 35.01 \\
\hline & & $35^{\circ} \mathrm{C}$ & & \\
\hline 0 & 0 & 62.07 & 1.1015 & 38.76 \\
\hline 20 & 0.175 & 63.92 & 1.0647 & 38.08 \\
\hline 40 & 0.362 & 65.98 & 1.0332 & 37.38 \\
\hline 60 & 0.561 & 68.17 & 1.0001 & 36.60 \\
\hline 80 & 0.744 & 70.51 & 0.9685 & 35.78 \\
\hline 100 & 1.000 & 73.14 & 0.9343 & 34.92 \\
\hline
\end{tabular}

Values in parenthesis were taken from ref. [16]. here the subscript " $s$ " and " $R$ " refer to the co-solvent (ethylene glycol and $\mathrm{N}, \mathrm{N}$-dimethyl formamide) mixtures and reference solvent (ethylene glycol) respectively and $M$ is the molar mass of the pure and mixed solvent. $\Delta G_{t}^{0}(i)$ and $T \Delta S_{t}^{0}(i)$ values of amino acids thus obtained and presented in the Table 3. The estimated values shows an uncertainties in $\Delta G_{t}^{0}(i)$ and $\Delta S_{t}^{0}(i)$ are about $\pm 0.05 \mathrm{~kJ} \cdot \mathrm{mol}^{-1}$ and $2 \mathrm{~kJ}^{-1} \cdot \mathrm{mol}^{-1}$, respectively.

\subsection{Computation of Chemical Part of Transfer Free Energy and Entropy}

Now $\Delta X_{t}^{0}(i)$ (where $X=G$ or $S$ ) may be ascribed as the sum of the following terms (assuming dipole induced dipole term to be negligibly small). i.e.

$$
\Delta X_{t}^{0}(i)=\Delta X_{t, c a v}^{0}(i)+\Delta X_{t, d d}^{0}(i)+\Delta X_{t, c h}^{0}(i)
$$

here, $\Delta X_{t, c a v}^{0}(i)$ means for the transfer energy contribution of the cavity effect which is involved due to creation of cavities for the species in ethylene glycol and ethylene glycol and $\mathrm{N}, \mathrm{N}$-dimethyl formamide mixed solvent system and $\Delta X_{t, d d}^{0}(i)$ stands for the dipole-dipole interaction effect involving interaction between dipolar-zwitter-ionic amino acids and the solvent molecules, on the other hand, $\Delta X_{c h}^{0}(i)$ includes that for all other effects such as those arising from acid-base or shortrange dispersion interaction, solvophilic or solvophobic solvation and structural effects etc. Here $\Delta X_{t, c a v}^{0}(i)$ values were computed by using Scaled particle theory (SPT) [17], assuming the solutes and solvent molecules as equivalent to hard-sphere models as dictated by their respective diameter (Vide Table 4).

$$
\Delta G_{t, d d}^{0}(i)=\left({ }_{s} \Delta G_{d d}^{0}(i)-{ }_{R} \Delta G_{d d}^{0}(i)\right)
$$

and

$$
\Delta S_{t, d d}^{0}(i)=\left({ }_{s} \Delta S_{d d}^{0}(i)-{ }_{R} \Delta S_{d d}^{0}(i)\right)
$$

were calculated by means of the Keesom-orientation expression [22] for ${ }_{s} \Delta G_{d d}^{0}(i)$ in a solvent $S$, as given below

$$
{ }_{s} \Delta G_{d d}^{0}(i)=-(8 \Pi / 9) N^{2} \mu_{s}^{2} \mu_{x}^{2} \sigma_{s x}^{-3}(k T)^{-1} v_{s}^{-1}=A / T V_{s}
$$

where

$$
A=-(8 \Pi / 9) N^{2} \mu_{s}^{2} \mu_{x}^{2} \sigma_{s x}^{-3}(k)^{-1}
$$

and

$$
V_{s}=M_{s} / d_{s}
$$

and that of $\Delta S_{d d}^{0}(i)$ as follows:

$$
{ }_{s} \Delta S_{d d}^{0}(i)=\left\{\delta_{s} \Delta G_{d d}^{0}(i) / \delta T\right\}_{p}
$$

i.e. $T_{s} \Delta S_{d d}^{0}(i)={ }_{s} \Delta G_{d d}^{0}(i)[1+T \alpha]$, where $N$ stands for Avogadro's number, $\mu_{s}, \mu_{x}$ are the dipole moment of 
Table 2. Solubilities (m) of Glycine, Dl-alanine, Dl- $\alpha$-amino butyric acid and Dl-nor-valine in binary mixtures of ethylene glycol and $N, N$-dimethyl formamide at different temperatures.

\begin{tabular}{|c|c|c|c|c|c|c|c|c|c|c|}
\hline \multicolumn{6}{|c|}{ Glycine } & \multicolumn{5}{|c|}{ Dl-alanine } \\
\hline $\mathrm{wt} \%$ solvent & $15^{\circ}$ & $20^{\circ}$ & $25^{\circ}$ & $30^{\circ}$ & $35^{\circ}$ & $15^{\circ}$ & $20^{\circ}$ & $25^{\circ}$ & $30^{\circ}$ & $35^{\circ}$ \\
\hline \multirow[t]{2}{*}{$100 \% \mathrm{EG}$} & 0.143 & 0.16 & 0.176 & 0.184 & 0.190 & 0.130 & 0.145 & 0.150 & 0.159 & 0.162 \\
\hline & $(0.143)^{\mathrm{a}}$ & & $(0.170)^{\mathrm{a}}$ & & $(0.186)^{\mathrm{a}}$ & $(0.126)^{\mathrm{a}}$ & \multicolumn{3}{|c|}{$(0.140)^{\mathrm{a}}$} & $(0.160)^{\mathrm{a}}$ \\
\hline $20 \% \mathrm{DMF}$ & 0.098 & 0.11 & 0.130 & 0.140 & 0.146 & 0.097 & 0.105 & 0.113 & 0.120 & 0.128 \\
\hline $40 \% \mathrm{DMF}$ & 0.069 & 0.077 & 0.084 & 0.093 & 0.101 & 0.078 & 0.084 & 0.090 & 0.096 & 0.102 \\
\hline $60 \% \mathrm{DMF}$ & 0.064 & 0.074 & 0.08 & 0.087 & 0.094 & 0.0603 & 0.064 & 0.0694 & 0.0738 & 0.078 \\
\hline $80 \% \mathrm{DMF}$ & 0.061 & 0.069 & 0.078 & 0.083 & 0.09 & 0.049 & 0.054 & 0.059 & 0.064 & 0.069 \\
\hline $100 \% \mathrm{DMF}$ & 0.038 & 0.042 & 0.046 & 0.049 & 0.052 & 0.041 & 0.044 & 0.048 & 0.0518 & 0.055 \\
\hline \multicolumn{6}{|c|}{ Dl-amino butyric acid } & \multicolumn{5}{|c|}{ D1-nor-valine } \\
\hline $\mathrm{wt} \%$ solvent & $15^{\circ}$ & $20^{\circ}$ & $25^{\circ}$ & $30^{\circ}$ & $35^{\circ}$ & $15^{\circ}$ & $20^{\circ}$ & $25^{\circ}$ & $30^{\circ}$ & $35^{\circ}$ \\
\hline \multirow[t]{2}{*}{$100 \% \mathrm{EG}$} & 0.200 & 0.212 & 0.224 & 0.240 & 0.254 & 0.118 & 0.128 & 0.139 & 0.156 & 0.160 \\
\hline & $(0.190)^{\mathrm{a}}$ & & $(0.220)^{\mathrm{a}}$ & & $(0.255)^{\mathrm{a}}$ & $(0.120)^{\mathrm{a}}$ & & $(0.130)^{\mathrm{a}}$ & & $(0.138)^{\mathrm{a}}$ \\
\hline $20 \% \mathrm{DMF}$ & 0.145 & 0.155 & 0.169 & 0.180 & 0.189 & 0.094 & 0.105 & 0.118 & 0.128 & 0.139 \\
\hline $40 \% \mathrm{DMF}$ & 0.102 & 0.110 & 0.120 & 0.126 & 0.1345 & 0.068 & 0.074 & 0.083 & 0.091 & 0.099 \\
\hline $60 \% \mathrm{DMF}$ & 0.079 & 0.086 & 0.094 & 0.099 & 0.104 & 0.039 & 0.048 & 0.052 & 0.057 & 0.062 \\
\hline $80 \% \mathrm{DMF}$ & 0.040 & 0.044 & 0.049 & 0.055 & 0.061 & 0.026 & 0.030 & 0.035 & 0.041 & 0.046 \\
\hline $100 \% \mathrm{DMF}$ & 0.036 & 0.040 & 0.045 & 0.050 & 0.056 & 0.022 & 0.026 & 0.032 & 0.036 & 0.042 \\
\hline
\end{tabular}

${ }^{\mathrm{a}}$ Ref. [17].

solvents and amino acid molecules respectively (Table 4).

$\sigma_{s x}$ is the distance at which the attractive and repulsive interactions between the solvent and solute molecules are equal and is generally equal to $1 / 2\left(\sigma_{s}+\sigma_{x}\right)$ where $\sigma_{s}$ and $\sigma_{x}$ are the hard sphere diameter of solvent and solute molecules respectively (Table 4) and $\alpha$ is the isothermal expansibility of the solvent and given by $\left(\delta \ln V_{s} / \delta T\right)_{P}=-\left(\delta \ln d_{s} / \delta T\right)$. Following Marcus [22] and Kim et al. [23] in order to get these $\Delta X_{t, d d}^{0}(i)$ term on mole fraction scale the quantity was again multiplied by the term $X_{s 1}$.

$$
X_{s 1}=X_{s}\left(\mu_{s} / \sigma_{s}^{3}\right) /\left(\mu_{R} / \sigma_{R}^{3}\right)
$$

which is the real mole fraction contribution due to dipole-dipole interaction [22]. Subtraction of $\Delta X_{t, c a v}^{0}(i)$ and $\Delta X_{t, d d}^{0}(i)$ from the total we can get $\Delta X_{t, c h}^{0}(i)$ of amino acids. The values of $\Delta X_{t, c a v}^{0}(i), \Delta X_{t, d d}^{0}(i)$ and $\Delta X_{t, c h}^{0}(i)$ are presented in Table 4.

\section{DISCUSSION}

\subsection{Type of Interactions of Amino Acids with Solvent Mixture}

The solubilities data reveals that the solubility of amino acids increases with the increase in temperature. Dl- $\alpha$ amino butyric acid is somewhat more soluble in the ethylene glycol and $N, N$-dimethyl formamide solvent mixtures than Dl-alanine, which is contrary to the prediction based on the hydrophobic nature of these two compounds. This may be due to the relative law crystal lattice energy of Dl- $\alpha$-amino butyric acid.

Figure 1 presents the variation of $\Delta G_{t}^{0}(i)$ of amino acids with mole $\% N, N$-dimethyl formamide in ethylene glycol and $N, N$-dimethyl formamide mixtures. $\Delta G_{t}^{0}(i)$ Values of four amino acids (i.e. Glycine, DL-alanine, DL- $\alpha$-amino butyric acid and DL-nor-valine) indicate their more or less gradual destabilizations with gradual increased concentration of $\mathrm{N}, \mathrm{N}$-dimethyl formamide in ethylene glycol and $\mathrm{N}, \mathrm{N}$-dimethyl formamide mixtures. As $\Delta G_{t}^{0}(i)$ is composed of $\Delta G_{t, c u v}^{0}(i), \Delta G_{t, d d}^{0}(i)$ and $\Delta G_{t, c h}^{0}(i)$ or others so their collective contribution to $\Delta G_{t}^{0}(i)$ show such little complex nature of variation with mole $\%$ of $N, N$-dimethyl formamide.

The upward trends of $\Delta G_{t, c h}^{0}(i)$ profiles of all amino acids (Figure 2) indicate their relative destabilization with increased concentration of $N, N$-dimethyl formamide. The order of stability with respect to chemical contribution of solute-solvent interaction is Dl-val. > Dl-Aba. > Dl-Ala. > Gly. 
Table 3. Coefficients a, b and c in Glycine, Dl-alanine, Dl- $\alpha$-amino butyric acid and Dl-norvaline and Gibbs energies $\Delta G_{t}^{0}$ and entropies $T \Delta S_{t}^{0}$ of transfer of the acids (on mole fraction scale) in $\mathrm{kJ} \cdot \mathrm{mol}^{-1}$ from Ethylene Glycol to Ethylene Glycol and $N, N$-dimethyl formamide mixtures at $25^{\circ} \mathrm{C}$.

\begin{tabular}{|c|c|c|c|c|c|}
\hline Solvents & $\begin{array}{c}\mathrm{a} \\
\left(\mathrm{kJ} \cdot \mathrm{mol}^{-1}\right)\end{array}$ & $\begin{array}{c}\mathrm{b} \\
\left(\mathrm{kJ} \cdot \mathrm{mol}^{-1} \cdot \mathrm{K}^{-1}\right)\end{array}$ & $\begin{array}{c}\mathrm{c} \\
\left(\mathrm{kJ} \cdot \mathrm{mol}^{-1} \cdot \mathrm{K}^{-1}\right)\end{array}$ & $\begin{array}{c}\Delta G_{t}^{0} \\
\left(\mathrm{~kJ} \cdot \mathrm{mol}^{-1}\right)\end{array}$ & $\begin{array}{c}T \Delta S_{t}^{0} \\
\left(\mathrm{~kJ} \cdot \mathrm{mol}^{-1}\right)\end{array}$ \\
\hline \multicolumn{6}{|c|}{ Glycine } \\
\hline $100 \% \mathrm{EG}$ & 255.67 & -5.5316 & 0.82289 & 0 & 0 \\
\hline $20 \% \mathrm{DMF}$ & 290.02 & -6.2071 & 0.92171 & 0.746 & 4.141 \\
\hline $40 \% \mathrm{DMF}$ & 35.87 & -0.5172 & 0.07326 & 1.667 & 2.036 \\
\hline $60 \% \mathrm{DMF}$ & 147.78 & -3.0374 & 0.44978 & 1.707 & 1.646 \\
\hline $80 \% \mathrm{DMF}$ & 167.03 & -3.4608 & 0.51282 & 1.725 & 2.082 \\
\hline $100 \% \mathrm{DMF}$ & 124.76 & -2.5575 & 0.37993 & 2.938 & -1.779 \\
\hline \multicolumn{6}{|c|}{ Dl-alanine } \\
\hline $100 \mathrm{EG}$ & 101.00 & -2.1190 & 0.31519 & 0 & 0 \\
\hline $20 \% \mathrm{DMF}$ & 32.66 & -0.5185 & 0.07496 & 0.688 & 2.596 \\
\hline $40 \% \mathrm{DMF}$ & 36.01 & -0.6055 & 0.08859 & 1.174 & 1.396 \\
\hline $60 \% \mathrm{DMF}$ & 26.46 & -0.3869 & 0.05622 & 1.731 & 0.941 \\
\hline $80 \% \mathrm{DMF}$ & 51.20 & -0.8859 & 0.12948 & 2.060 & 3.509 \\
\hline $100 \% \mathrm{DMF}$ & 20.34 & -0.2177 & 0.03067 & 2.481 & 1.689 \\
\hline \multicolumn{6}{|c|}{ Dl- $\alpha$-aminobutyric acid } \\
\hline $100 \% \mathrm{EG}$ & -36.37 & 0.9983 & -0.15163 & 0 & 0 \\
\hline $20 \% \mathrm{DMF}$ & 64.87 & -1.2511 & 0.18400 & 0.656 & 0.516 \\
\hline $40 \% \mathrm{DMF}$ & 63.49 & -1.2145 & 0.17889 & 1.429 & -0.114 \\
\hline $60 \% \mathrm{DMF}$ & 184.26 & -3.9182 & 0.58267 & 1.927 & -0.217 \\
\hline $80 \% \mathrm{DMF}$ & -36.58 & 1.1475 & -0.17548 & 3.443 & 3.458 \\
\hline $100 \% \mathrm{DMF}$ & -10.72 & 0.5762 & -0.0903 & 3.578 & 3.787 \\
\hline \multicolumn{6}{|c|}{ Dl-nor-valine } \\
\hline $100 \% \mathrm{EG}$ & -100.62 & 2.5476 & -0.38504 & 0 & 0 \\
\hline $20 \% \mathrm{DMF}$ & 100.79 & -1.970 & 0.28963 & 0.383 & -0.126 \\
\hline $40 \% \mathrm{DMF}$ & 15.67 & -0.0609 & 0.00511 & 1.177 & -1.210 \\
\hline $60 \% \mathrm{DMF}$ & 239.59 & -5.0502 & 0.74963 & 2.201 & -0.293 \\
\hline $80 \% \mathrm{DMF}$ & 27.54 & -0.1811 & 0.02045 & 3.103 & 4.160 \\
\hline $100 \% \mathrm{DMF}$ & 96.06 & -1.6717 & 0.24193 & 3.787 & 6.402 \\
\hline
\end{tabular}

The size of $N, N$-dimethyl formamide $(0.498 \AA)$ is greater than ethylene glycol $(0.437 \AA)$. Thus free energy change due to cavity formation is also more negative in $\mathrm{N}, \mathrm{N}$-dimethyl formamide relative to ethylene glycol. $\Delta G_{t, c a v}^{0}(i)$ is more negative for the higher homologue among four amino acids having larger hard-sphere di- ameter (Table 4). On the other hand the dipole moment of $\mathrm{N}, \mathrm{N}$-dimethyl formamide (3.82D) is also greater than ethylene glycol (2.28D). Therefore, $\Delta G_{t, d d}^{0}(i)$ values are more negative in higher concentration of $N, N$-dimethyl formamide in this ethylene glycol and $N, N$-dimethyl formamide mixed solvent system. The order of $\Delta G_{t, d d}^{0}(i)$ is 
Table 4. Gibbs energies of transfer $\Delta G_{t}^{0}(i), \Delta G_{t, c a v}^{0}(i), \Delta G_{t, d d}^{0}(i), \Delta G_{t, c h}^{0}(i)$ and enthalpy of transfer, $\Delta H_{t, c a v}^{0}(i)$ and entropies of transfer $T \Delta S_{t}^{0}(i), T \Delta S_{t, c a v}^{0}(i), T \Delta S_{t, d d}^{0}(i)$ and $T \Delta S_{t, c h}^{0}(i)$ of Glycine, Dl-alanine, Dl- $\alpha$-amino-butyric acid and Dl-nor-valine from Ethylene Glycol to Ethylene Glycol and $N, N$-dimethyl formamide mixtures at $25^{\circ} \mathrm{C}$ (on mole fraction scale in $\mathrm{kJ} \cdot \mathrm{mol}^{-1}$ ).

\begin{tabular}{|c|c|c|c|c|c|c|c|c|c|}
\hline Solvents & $\Delta G_{t}^{0}(i)$ & $\Delta G_{t, c a v}^{0}(i)$ & $\Delta G_{t, d d}^{0}(i)$ & $\Delta G_{t, c h}^{0}(i)$ & $T \Delta S_{t}^{0}(i)$ & $\Delta H_{t, c a v}^{0}(i)$ & $T \Delta S_{t, c a v}^{0}(i)$ & $T \Delta S_{t, d d}^{0}(i)$ & $T \Delta S_{t, c h}^{0}(i)$ \\
\hline \multicolumn{10}{|c|}{ Glycine } \\
\hline $100 \% \mathrm{EG}$ & 0 & 0 & 0 & 0 & 0 & 0 & 0 & 0 & 0 \\
\hline $20 \% \mathrm{DMF}$ & 0.746 & -0.495 & -1.13 & 2.371 & 4.141 & -0.324 & 0.171 & -1.53 & 5.50 \\
\hline $40 \% \mathrm{DMF}$ & 1.667 & -0.925 & -4.95 & 7.542 & 2.036 & -0.112 & 0.813 & -6.80 & 8.023 \\
\hline $60 \% \mathrm{DMF}$ & 1.707 & -1.291 & -11.60 & 14.598 & 1.646 & 0.754 & 2.045 & -16.30 & 15.901 \\
\hline $80 \% \mathrm{DMF}$ & 1.725 & -1.578 & -21.80 & 25.103 & 2.082 & 2.597 & 4.175 & -31.30 & 29.207 \\
\hline $100 \% \mathrm{DMF}$ & 2.938 & -1.762 & -35.50 & 40.20 & -1.779 & 6.062 & 7.824 & -52.10 & 42.522 \\
\hline \multicolumn{10}{|c|}{ Alanine } \\
\hline $100 \% \mathrm{EG}$ & 0 & 0 & 0 & 0 & 0 & 0 & 0 & 0 & 0 \\
\hline $20 \% \mathrm{DMF}$ & 0.688 & -0.523 & -1.01 & 2.221 & 2.596 & -0.379 & 0.144 & -1.36 & 3.812 \\
\hline $40 \% \mathrm{DMF}$ & 1.174 & -0.969 & -4.42 & 6.563 & 1.396 & -0.140 & 0.829 & -6.07 & 6.637 \\
\hline $60 \% \mathrm{DMF}$ & 1.731 & -1.343 & -10.30 & 13.363 & 0.746 & 0.851 & 2.194 & -14.40 & 12.952 \\
\hline $80 \% \mathrm{DMF}$ & 2.060 & -1.627 & -19.60 & 23.287 & 3.503 & 2.966 & 4.593 & -28.01 & 26.92 \\
\hline $100 \% \mathrm{DMF}$ & 2.481 & -1.794 & -31.90 & 36.135 & 1.106 & 6.944 & 8.738 & -46.80 & 39.751 \\
\hline \multicolumn{10}{|c|}{ Amino-butyric acid } \\
\hline $100 \% \mathrm{EG}$ & 0 & 0 & 0 & 0 & 0 & 0 & 0 & 0 & 0 \\
\hline $20 \% \mathrm{DMF}$ & 0.656 & -0.546 & -0.937 & 2.139 & 0.516 & -0.427 & 0.119 & -1.26 & 1.657 \\
\hline $40 \% \mathrm{DMF}$ & 1.429 & -1.008 & -4.01 & 6.447 & -0.114 & -0.165 & 0.843 & -5.51 & 4.533 \\
\hline $60 \% \mathrm{DMF}$ & 1.927 & -1.387 & -9.44 & 12.754 & -0.217 & 0.934 & 2.321 & -13.2 & 10.662 \\
\hline $80 \% \mathrm{DMF}$ & 3.443 & -1.668 & -18.0 & 23.111 & 3.458 & 3.282 & 4.950 & -25.7 & 24.208 \\
\hline $100 \% \mathrm{DMF}$ & 3.578 & -1.821 & -29.30 & 34.699 & 3.787 & 7.700 & 9.521 & -42.80 & 37.066 \\
\hline \multicolumn{10}{|c|}{ Nor-valine } \\
\hline $100 \% \mathrm{EG}$ & 0 & 0 & 0 & 0 & 0 & 0 & 0 & 0 & 0 \\
\hline $20 \% \mathrm{DMF}$ & 0.383 & -0.566 & -0.860 & 1.809 & -0.126 & 0.680 & 1.246 & -1.16 & 0.212 \\
\hline $40 \% \mathrm{DMF}$ & 1.177 & -1.040 & -3.69 & 5.907 & -1.210 & 0.961 & 2.001 & -5.06 & 1.849 \\
\hline $60 \% \mathrm{DMF}$ & 2.201 & -1.424 & -8.69 & 12.332 & -0.293 & 2.150 & 3.574 & -12.20 & 8.333 \\
\hline $80 \% \mathrm{DMF}$ & 3.103 & -1.703 & -16.50 & 20.745 & 4.160 & 4.696 & 6.399 & -23.60 & 21.361 \\
\hline $100 \% \mathrm{DMF}$ & 3.347 & -1.845 & -27.00 & 31.89 & 6.402 & 9.487 & 11.332 & -39.50 & 34.57 \\
\hline
\end{tabular}

The required diameter and other solvent parameters of ethylene glycol and $N, N$-dimethyl formamide mixtures are taken from Ref. [22].The required diameter of glycine, alanine, amino butyric acid and nor-valine are 5.64, 6.16,6.58 and $6.92 \AA$, respectively, as given in Ref. [24]. Dipole-moment values of $\alpha$-amino acids are 15.7D for glycine, 15.9D for alanine and 16D for amino butyric acid and nor-valine [25].

Gly. > Dl-Ala. > Dl-Aba. > Dl-nor-val. As $\Delta G_{t, d d}^{0}(i)$ values are guided by dipole moment and hard-sphere diameter of both solutes (here amino acid) as well as solvent, the above order is well supported from Table 4.

$\Delta G_{t, c h}^{0}(i)$ values of the four amino acid represent the free energy change in the ethylene glycol and $N, N$-dimethyl formamide mixed solvent system due to different short range chemical interactions i.e. acid-base, dispersion, hard-soft, H-bonding, solvophilic/solvophobic interaction etc. 


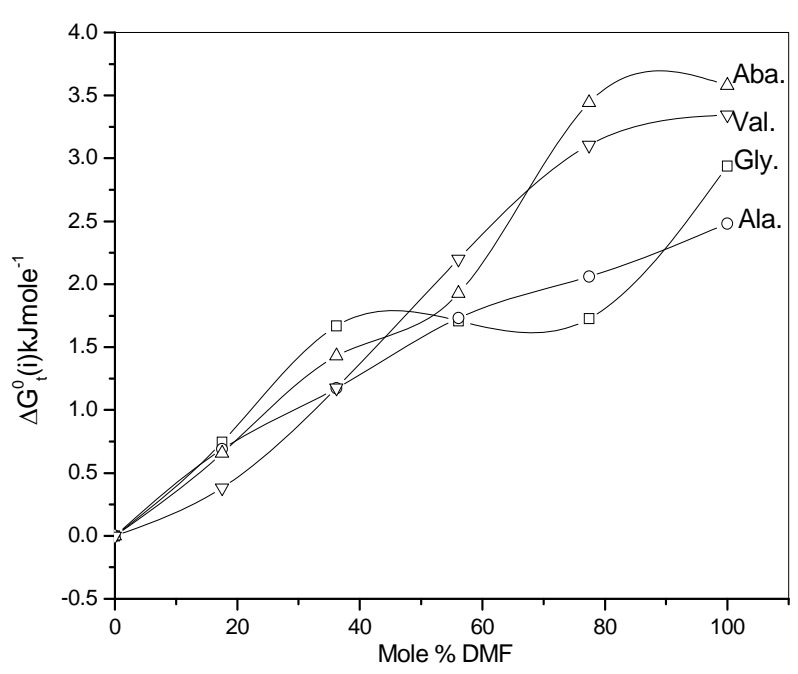

Figure 1. Variation of $\Delta G_{t}^{0}(i) \mathrm{kJ} \cdot \mathrm{mol}^{-1}$ of glycine, Dl-alanine, Dl-alpha amino butyric acid and Dl-nor-valine in non-aqueous ethylene glycol and $N, N$-dimethyl formamide mixtures.

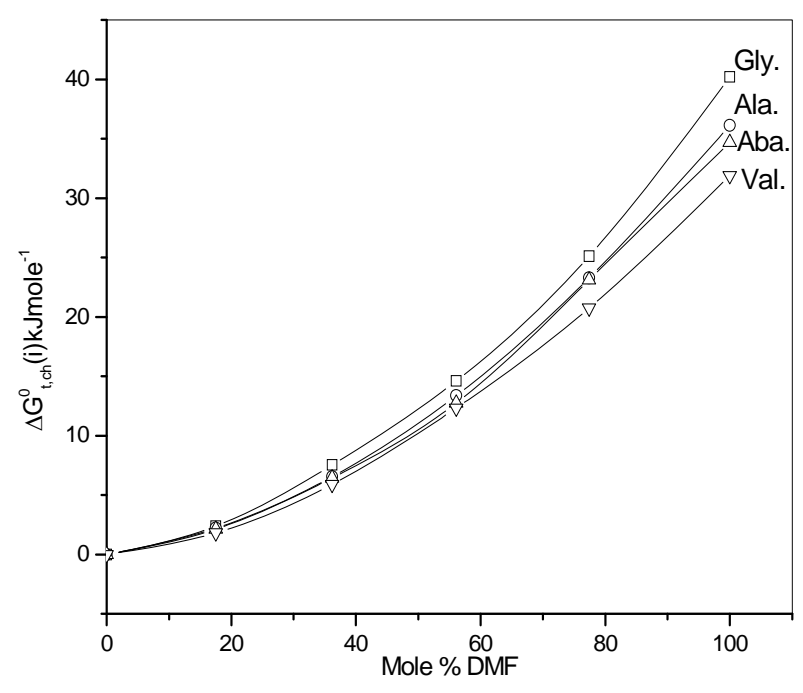

Figure 2. Variation of $\Delta G_{t, c h}^{0}(i) \mathrm{kJ} \cdot \mathrm{mol}^{-1}$ of glycine, Dlalanine, Dl-alppha amino butyric acid and Dl-nor valine in non-aqueous ethylene glycol and $N, N$-dimethyl formamide mixture.

As the proportion of $\mathrm{N}, \mathrm{N}$-dimethyl formamide in the mixed solvent system will be gradually increased the solvent character may undergoes a gradual but material change in respect to the above types of chemical interactions.

While ethylene glycol is a good Bronsted acid, $\mathrm{N}, \mathrm{N}$-dimethyl formamide is not. Thereby anionic part $\left(\mathrm{COO}^{-}\right)$of these four amino acids can be more solvated in ethylene glycol than $\mathrm{N}, \mathrm{N}$-dimethyl formamide due to acid-base interaction. Therefore with the increased concentration of $N, N$-dimethyl formamide $\Delta G_{t, c h}^{0}(i)$ values become more and more positive. On the other hand the cationic part $\left(\mathrm{NH}_{3}^{+}\right)$of the zwitterionic form of amino acids will be more solvated with the increased concentration of $N, N$-dimethyl formamide as it possess stronger lewis basicity and cationophilicity than ethylene glycol. Furthermore, in respect of H-bonding capacity ethylene glycol is more potential than $\mathrm{N}, \mathrm{N}$-dimethyl formamide. So amino acids will be less solvated with increased concentration of $N, N$-dimethyl formamide in ethylene glycol and $N, N$-dimethyl formamide mixtures.

It should be noted that $N, N$-dimethyl formamide $(0.498$ $\AA)$ is more polarisable than ethylene glycol $(0.437 \AA)$. Therefore $N, N$-dimethyl formamide, here undergo more stronger soft-soft and dispersion interactions with larger amino acids (i.e. $\alpha$-aba $\& \mathrm{n}$-val).These phenomenon is well evidenced in the stability order i.e. $\Delta G_{t, c h}^{0}(i)$ (n-val.) $>\Delta G_{t, c h}^{0}(i) \quad(\alpha$-aba. $)>\Delta G_{t, c h}^{0}(i) \quad($ ala. $) \stackrel{>}{>} \Delta G_{t, c h}^{0}(i)$ (gly.).

For larger amino acids like Dl-alanine, Dl- $\alpha$-amino butyric acid and Dl-nor-valine along with soft-soft, dispersion and specific charge transfer interactions and another indirect and hence secondary interaction, namely solvophilic solvation $\left(\mathrm{S}_{b} \mathrm{~S}\right)$ is likely to be significant. Ethylene glycol, like $\mathrm{H}_{2} \mathrm{O}$, being extensively capable of intermolecular hydrogen bond formation, organizes a cage-like structure around organic moiety [here $-\mathrm{CH}_{3}$ (Alanine), $-\mathrm{CH}_{3}-\mathrm{CH}_{3}$ ( $\alpha$-amino butyric acid), $-\mathrm{CH}_{3}-$ $\mathrm{CH}_{3}-\mathrm{CH}_{3}$ (non-valine)] by being induced by the latter, causing 'solvophilic solvation' which is similar to 'hydrophobic hydration' $\left(\mathrm{H}_{\mathrm{b}} \mathrm{H}\right)$ in aqueous solution.

Thus for larger amino acids although dispersion interaction is significant and tends to decrease $\Delta G_{t, c h}^{0}(i)$ values, the latter "solvophilic salvation" $\left(\mathrm{S}_{\mathrm{b}} \mathrm{S}\right)$ still decrease and tends to increases $\Delta G_{t, c h}^{0}(i)$ values with increase concentration of DMF as compared to those in pure ethylene glycol.

Thus the chemical contribution of transfer free energies, $\Delta G_{t, c h}^{0}(i)$ of these four homologous $\alpha$-amino acids are guided by the composite effects of increased dispersion interaction, basicity and decreased acidity, hydrogen bonding effects and solvophobic solvation of ethylene glycol and $N, N$-dimethyl formamide mixtures as compared to that of reference solvent (ethylene glycol).

\subsection{Role of Amino Acids for Controlling Solvent-Solvent Interactions}

Figure 3 represents the $T \Delta S_{t}^{0}(i)$ change of these amino acids with ethylene glycol and $N, N$-dimethyl formamide mixtures. Here for all four amino acids rollercoaster type behavior are found. Like $\Delta G_{t}^{0}(i), T \Delta S_{t}^{0}(i)$ can be taken to be composed of cavity, dipole-dipole and chemical interaction effects i.e. 


$$
T \Delta S_{t}^{0}(i)=T \Delta S_{t, c a v}^{0}(i)+T \Delta S_{t, d d}^{0}(i)+T \Delta S_{t, c h}^{0}(i)
$$

where $T \Delta S_{t, c a v}^{0}(i)$ represents the difference of entropy change involved in creating appropriate cavities for accommodating the amino acids molecule in the reference solvent ethylene glycol and ethylene glycol and $\mathrm{N}, \mathrm{N}$ dimethyl formamide mixed co-solvent system in the present study.

$T \Delta S_{t, d d}^{0}(i)$ stands for the dipole-dipole interactions originated due to dipolar amino acid molecule and dipolar mixed solvent system. $T \Delta S_{t, c h}^{0}(i)$ term referred to as the chemical effect, stands for the structural interaction effect that appears due to the change of solvent structure induced by the amino acid molecules, if any, apart from that involved in the cavity effect. Now combined effect in $T \Delta S_{t}^{0}(i)$ values may represents such behavior as shown in Figure 3.

In Figure $4 T \Delta S_{t, c h}^{0}(i)$ Vs ethylene glycol and $N, N$ dimethyl formamide mixed solvent composition profiles is illustrated. For all amino acids regular increase of $T \Delta S_{t, c h}^{0}(i)$ values with increase concentration of of $N, N$ dimethyl formadide is reflected. The $T \Delta S_{t, c h}^{0}(i)$ value varies as Dl-n val. $<$ Dl- $\alpha$-aba. $<$ Dl-ala. $<$ gly. Now in order to understand the variation of $T \Delta S_{t, c h}^{0}(i)$ for all four amino acids with increased DMF concentration in ethylene glycol and $N, N$-dimethyl formamide mixed solvent systems; one must note that solvation here occurs mainly through solvophobic solvation $\left(\mathrm{S}_{\mathrm{b}} \mathrm{S}\right)$, a phenomenon similar to hydrophobic hydration $\left(\mathrm{H}_{b} \mathrm{H}\right)$ as in aqua-organic systems.

Solvation by this effect, solphobic solvation $\left(\mathrm{S}_{\mathrm{b}} \mathrm{S}\right)$ significantly decreases the entropy in ethylene glycol. Gradual desolvation with increased concentration of $N, N$-dimethyl formadide in ethylene glycol and $N, N$ dimethyl formamide mixtures for these amino acids is also guided by the increased hard-sphere diameter (Dl-n val. $>$ Dl- $\alpha$-aba. $>$ Dl-ala. $>$ gly.) of the amino acids.

Therefore as reflected in Figure 4, Dl-nor-valine will be more desolvated (i.e. lower $T \Delta S_{t, c h}^{0}$ ) than other and glycine will be least desolvated (i.e. higher $T \Delta S_{t, c h}^{0}$ ).

On the other hand observed monotonic increase of $T \Delta S_{t, c h}^{0}$ for these amino acids along with other interactions, is also guided by dispersion interaction. But solvophobic solvation being larger in the reference solvent, ethylene glycol than in the mixed solvents, it is quite likely that transfer of amino acids will disrupt the solvent structure, and hence make $T \Delta S_{t, c h}^{0}$ (amino acids) increasingly positive, as observed in Figure 4.

\section{CONCLUSIONS}

From the overall observations it may be concluded that the stability of the four homologous $\alpha$-amino acids are guided by superimposed effects of increased cavity

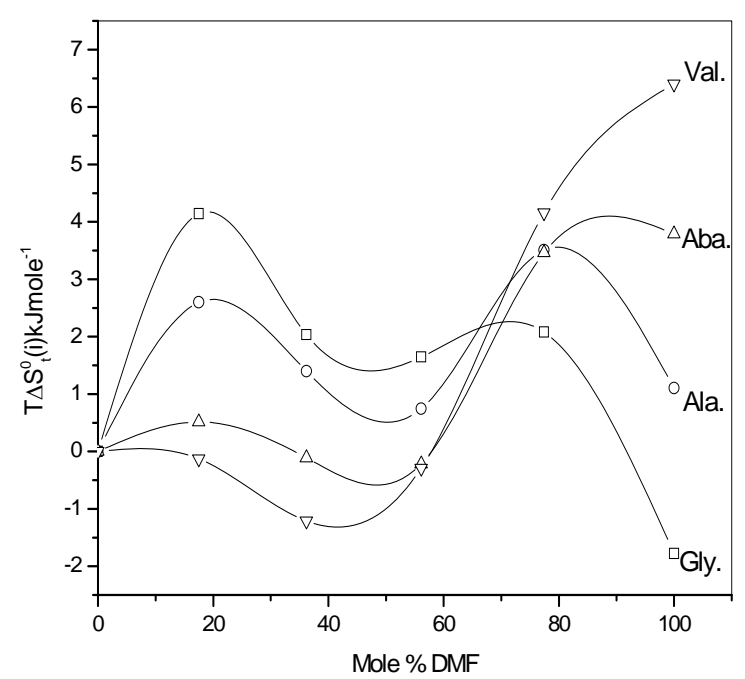

Figure 3. Variation of $T \Delta S_{t}^{0}(i)$ of glycine, Dl-alanine, Dl-alpha amino butyric acid and Dl-nor-valine in non aqueous ethylene glycol and $N, N$-dimethyl formamide mixtures.

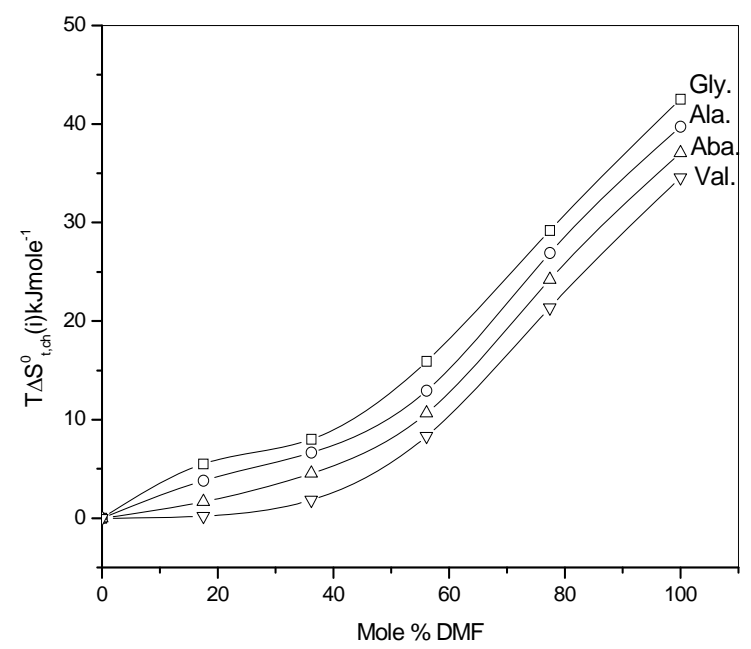

Figure 4. Variation of $T \Delta S_{t, c h}^{0}(i)$ of glycine, Dl-alanine, Dl-alpha amino butyric acid and Dl-nor-valine innon-aqueous ethylene glycol and $N, N$-dimethyl formamide mixtures.

effect, dipole-dipole interactions, dispersion interactions, basicity effect and decreased acidity effect, solvophovic solvation with increased concentrations of $\mathrm{N}, \mathrm{N}$-dimethyl formamide in ethylene glycol and $N, N$-dimethyl formamide mixtures. Also it is transpiring that ethylene glycol, having protic character will be good stabilizer of amino acids, proteins as well as dipolar biomolecules. On the other hand, dipolar aprotic $N, N$-dimethyl formamide solvent will be good stabilizer for heavier amino acids having larger apolar moieties. Structural eccentricities of $\mathrm{H}_{2} \mathrm{O}$ solvent may also be indirectly reestablished here by quite different type of solute-solvent interactional behaviour in our present solvent system. 


\section{ACKNOWLEDGEMENTS}

The authors record their kind thanks to DST-SAP, UGC, Govt. of India and the Dept. of chemistry, Visva-Bharati for financial assistance and computational facilities.

\section{REFERENCES}

[1] Das, P., chatterjee, S. and Basu Mallick, I. (2004) Thermodynamic studies on amino acid solvation in some aqueous alcohols. Journal of Chinese Chemical Society, 51, 1-6.

[2] Banipal, T.S., Singh. G. and Lark, B.S. (2001) Partial molar volumes of transfer of some amino acids from water to aqueous glycerol solutions at $25^{\circ} \mathrm{C}$. Journal of Solution Chemistry, 30, 657.

[3] Lapamje, S. (1978) In physico-chemical aspects of proteins denaturation. Wiley Intercience, New York, 241.

[4] Islam, M.N. and Wadi, R.K. (2001) Thermodynamics of transfer of amino acids from water to aqueous sodium sulfate. Physics and Chemistry of Liquids, 39, 77-84. doi:10.1080/00319100108030328

[5] Köseoglu, F., Kiliçb, E. and Dogan, A. (2000) Studies on the protonation constants and solvation of $\alpha$-amino acids in dioxan-water mixtures. 277, 243-246.

[6] Anfinsen, C.B and Seheraga, H.A. (1978) Experimental and theoretical aspects of protein folding. Advances in Protein Chemistry, 29, 205-300. doi:10.1016/S0065-3233(08)60413-1

[7] Reading, J.F., Watson, I.D. and Gavin, R.H. (1990) Thermodynamic properties of peptide solutions 5. Partial molar volumes of glycylglycine, glycyl-DL-leucine, and glycyl-DL-serine at 308.15 and $318.15 \mathrm{~K}$. The Journal of Chemical Thermodynamics, 22, 159-165. doi:10.1016/0021-9614(90)90079-6

[8] Nozaki, Y. and Tanford, C. (1963) The solubilities of amino acids and related compounds in aqueous urea solutions. The Journal of Biological Chemistry, 238, 40744081 .

[9] Abu-Hamdiyyah, M. and Shehabuddin, A. (1982) Transfer enthalpies and entropies of amino acids from water to urea solutions. Journal of Chemical Engineering Data, 27, 74-76.

[10] Gekko, K. and Timasheff, S.N. (1981) Thermodynamic and kinetic examination of protein stabilization by glycerol. Biochemistry, 20, 4677. doi:10.1021/bi00519a024

[11] Sinha, R., Bhattacharya, S.K. and Kundu, K.K. (2005) Chemical transfer energetic of the $-\mathrm{CH}_{2}$-group in aqueous glycerol: Solvent effect on hydrophobic hydration and its three-dimensional structure. Journal of Molecular Liquids, 122, 95-103. doi:10.1016/j.molliq.2005.04.003

[12] Roy, S., Mahali. K. and Dolui, B.K. (2009) Thermodynamic studies of solvation a series of homologous $\alpha$-amino acids in aqueous mixtures of protic ethylene glycol at $298.15^{\circ}$ C. Biochemistry-An Indian Journal, 3, 63-68.
[13] Ganguly, S. and Kundu, K.K. (1993) Transfer energetic of some DNA and RNA bases in aqueous mixtures of urea and glycerol. Journal of Physical Chemistry, 97, 10862-10867. doi:10.1021/j100143a055

[14] Chatterjee, S. and Basumallick, I. (2007) Thermodynamic studies on amino acid solvation in aqueous urea. Journal of Chinese Chemical Society, 54, 1-6.

[15] Talukdar, H., Rudra, S.P. and Kundu, K.K. (1988) Thermodynamics of transfer of glycine, diglycine, and triglycine from water to aqueous solutions of urea, glycerol, and sodium nitrate. Canadian Journal of Chemistry, 66, 461-468. doi: 10.1139/v88-080

[16] Dolui, B.K., Bhattachary, S.K. and Kundu, K.K. (2006) Single-ion transfer Gibbs energies in binary mixtures of isodielectric protic ethylene glycol and dipolar aprotic $\mathrm{N}, \mathrm{N}$-dimethylformamide. Indian Journal of Chemistry, 45A, 2607-2614.

[17] Roy, S., Mahali, K. and Dolui. B.K. (2010) Tranfer entropies of solvation of a series of homologous $\alpha$-amino acids in aqueous mixtures of protic ethylene glycol. Biochemistry, An Indian Journal, 3, 71-76.

[18] Datta, J. and Kundu, K.K. (1982) Transfer thermodynamics of benzoic acid in aqueous mixtures of some ionic and nonionic co-solvent and the structuredness of solvents. Journal of Physical Chemistry, 86, 4055-4061. doi: $10.1021 / \mathrm{j} 100217 \mathrm{a} 034$

[19] Datta, J. and Kundu, K. K. (1983) Transfer thermodynamics of $p$-nitro aniline in aqueous solutions of some ionic and non-ionic co-solvents and the structuredness of the solvents. Canadian Journal of Chemistry, 61, 62.

[20] Majumder, K. and Lahiri, S.C. (1997) Studies on the dissociation constants and solubilities of amino acids in dioxane + water mixtures at 298.15 K. Journal of Indian Chemical Soceity, 74, 382.

[21] Dutta, S.C. and Lahiri, S.C. (1995) Studies on the dissociation constants and solubilities of amino acids in ethylene glycol + water mixtures. Journal of Indian Chemical Soceity, 72, 315.

[22] Marcus, Y. (1985) Ion solvation. John Wilcy and Sons, New York, 37.

[23] Kim, J.I., Cocal, A., Born, H. and Comma, E.A. (1978) Preferential salvation of ions: Acritical study of the $\mathrm{Ph}_{4} \mathrm{AsPh}_{4} \mathrm{~B}$ assumption for single ion thermodynamics in mixed aqueous-acetonitrile and aqueous- $N, N$-Dimethylformamide solvents. Zeitschrift fur Physikalische Chemie Neue Folge, 110, 209.

[24] Sinha, R. and Kundu, K.K. (2004) Transfer energetics of a series of homologous $\alpha$-amino acids and hence of $-\mathrm{CH}_{2}$-group - A possible probe for the solvent effect on hydrophobic hydration and the hence three dimensional-structuredness of aqueous cosolvents. Journal of Molecular Liquids, 111, 151-159. doi:10.1016/j.molliq.2003.12.015

[25] Hill, N.E., Baughan, W.E., Price, A.H. and Davics, M. (1969) Dielectric properties and moleculer behavior. Van Nostrand Reinhold Co., London, 480. 